\title{
Informing the Design of Situated Glyphs for a Care Facility
}

\author{
Jo Vermeulen*, Fahim Kawsar ${ }^{\dagger}$, Adalberto L. Simeone ${ }^{\ddagger}$, Gerd Kortuem ${ }^{\S}$, Kris Luyten*, and Karin Coninx* \\ ${ }^{*}$ Expertise Centre for Digital Media - Hasselt University - tUL - IBBT \\ Email: [jo.vermeulen, kris.luyten, karin.coninx] @uhasselt.be \\ ${ }^{\dagger}$ Bell Labs, Belgium \\ Email: fahim.kawsar@alcatel-lucent.com \\ ${ }^{\ddagger}$ School of Computing \& Communications, Lancaster University, UK \\ Email: a.simeone@lancaster.ac.uk

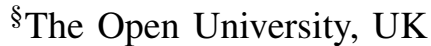 \\ Email: g.kortuem@open.ac.uk
}

\begin{abstract}
Informing caregivers by providing them with contextual medical information can significantly improve the quality of patient care activities. However, information flow in hospitals is still tied to traditional manual or digitised lengthy patient record files that are often not accessible while caregivers are attending to patients. Leveraging the proliferation of pervasive awareness technologies (sensors, actuators and mobile displays), recent studies have explored this information presentation aspect borrowing theories from context-aware computing, i.e., presenting subtle information contextually to support the activity at hand. However, the understanding of the information space (i.e., what information should be presented) is still fairly abstruse, which inhibits the deployment of such real-time activity support systems. To this end, this paper first presents situated glyphs, a graphical entity to encode situation specific information, and then presents our findings from an in-situ qualitative study addressing the information space tailored to such glyphs. Applying technology probes using situated glyphs and different glyph display form factors, the study aimed at uncovering the information space pertained to both primary and secondary medical care. Our analysis has resulted in a large set of information types as well as given us deeper insight on the principles for designing future situated glyphs. We report our findings in this paper that we expect would provide a solid foundation for designing future assistive systems to support patient care activities.
\end{abstract}

\section{INTRODUCTION}

Improving the quality of care is one of the fundamental strategies for a better health system. Various measures are being pursued by health institutions to reach this goal. For example, the UK government in 2004 introduced the "Pay for performance" incentive scheme, which linked approximately $25 \%$ of a physician's income to 146 quality indicators [1]. Similar measures are in effect in the US where these programs affect not only physicians but also hospitals. Initial reports on the effects of the adoption of these programs point out either plateaued [2] or modest increases in clinical performance [3].

Hospitals are dynamic environments where due to the high workload, it is not uncommon for certain events to go unnoticed [4]. For example, patients might lie in the same position for too long or they could have to wait longer than expected for a nurse to respond to an alarm call. These events can negatively affect how a patient believes she is being treated by the hospital. In light of this, context-aware computing - the potential of applications to present information that is relevant to the context of the users, such as location, time, and current activities [5], [6] - can intervene to provide support measures for both hospital staff and patients.

Recent work has investigated assistive system design aimed at capturing short term events as well as presenting patient records and other contextual information in graphical languages to improve patient care experience [4], [7]-[10]. In our previous work, we introduced the notion of situated glyphs to support real-time medical information provisioning using graphical representation [11]. In an hospital environment, there is an established ubiquity of graphical signs as they are used for a wide range of purposes: directions to specific areas of the ward, rules to abide by, status information on medical equipment, etc. However, these graphical representations are seldom dynamic in nature or context-aware as they are confined to printed material or desktop applications aimed at practitioners. The notion of situated glyphs stems from the implicit acceptance that they have in this particular workplace. In contrast to their static counterparts, the idea of displays presenting glyphs has the unique advantage of being able to portray information that can adapt to real time events and situations, thus being always up to date and current something that can have a profound impact on the quality of care. Furthermore, they do not require any radical changes in the current work practices of hospital staff.

However, the understanding of the medical information space that can be encoded into glyphs, i.e., the diversity of information, temporal priority, as well as proper presentation notation is still fairly poor, which in turn inhibits the successful deployment of such system. In this paper, we address this particular aspect of understanding the information space by reporting on a qualitative study where we exposed caregivers to the idea of situated glyphs. To uncover the information space, we conducted a workshop at a psychogeriatrics ward in the Netherlands, following a participatory design approach. As a technology probe, we have used a different set of glyph designs, slightly different information types and micro displays. This study gave us a deeper insight on the informa- 
tion space and resulted in identifying 28 unique information types. Furthermore, active participation from the caregivers in encoding this information allowed us to capture 54 design examples that can be represented with situated glyphs. Based on these findings we offer design guidelines for future assistive systems aimed at supporting healthcare environments.

\section{ReLATEd Work}

The literature presents several examples of pervasive systems in the medical domain. However, these works usually focus on single or specific tasks to improve. A wide range of different monitoring devices are commonly found in hospital rooms; these devices can be potentially used to provide various streams of data. For example, numerous efforts have been made to go beyond traditional audiovisual notifications: Favela et al. [10] present an activity recognition system that, by using a neural network, presents relevant information to the estimated activity being performed. Sensors can also be integrated seamlessly within the hospital environment: many object that are commonly available can be augmented to provide contextual information. Bardram [9] have augmented a bed and pill container with RFID tags. The bed is able to detect the patient lying on it and a nurse in its vicinity. An embedded touchscreen display reacts to the environment by suggesting the medicine schema to the nurse, assuring that the right one is chosen. The display device also acts as a normal PC screen allowing the patient to check her/his emails and watch TV. The pill container supports medicine administration by highlighting the correct tray holding the pills associated to a nearby patient. Large interactive displays can supersede traditional whiteboards to show surgery schedules and situational information about operating rooms [12]. Objects that are not traditionally seen as displays can also present information. Segura et al. [13] used a flower vase and a motion statue to convey patient status data. The first device has two sets of flowers that blink when a patient's urine bag is almost full and needs to be replaced. The first set of flowers is activated by a weight sensor connected to the bag. The second set of flowers, being arranged in a grid, gives the actual position of the interested patient. These augmented devices aim to support nurses in an unobtrusive way, so that their actual purpose can only be understood by those who are aware of their meaning.

In this way, these technologies tend to become a fundamental part of hospital processes. However, users acceptance of these systems is not obvious as some studies indicate that hospital staff may be wary of adopting certain technologies, because of the concern that they might also be employed for surveillance purposes [14], [15]. Hospitals are highly dynamic workplaces in which daily activities are inherently distributed over the physical environment. Hospital staff is therefore seldom tied in a fixed place, as they have to check multiple patient rooms, access different hospital facilities such as medicine rooms, operating theaters, etc. Errors during medical practice are unfortunately not rare: prescription errors were reported to be as high as $13 \%$ of the cases analysed in one study [16]. These errors are mostly due to nurses not being completely aware of what could already have been done by other staff.
Erroneously administered drugs can lead to life-threatening events.

In light of this, support for roaming activities, even through displays, has been proposed as a very important aspect in the design of hospital information systems [7]. In the literature, there is ample evidence of the power that graphical representations have to quickly convey information [17]. One such method is encoding data into glyphs, pictograms representing multivariate data values through different graphical attributes, e.g., shape, colour, size, etc. By themselves or in combination between each other, glyphs are able to communicate vast amounts of information. Glyphs have been used to encode data features in several works on information visualization. Ropinski and Preim explored their use in medical visualizations [18]. They propose a taxonomy grounded on the theory of perception and classify glyphs according to how they communicate information to the viewer. A series of guidelines are defined to help guide the design of such glyphs. In the medical domain, there is often the need to encode patient data in succinct form. Traditional textual representations take time to read and fully comprehend and in this context, where physicians may have to browse several such patient records, they are far from being an ideal solution. Lamy et al. [19] define a graphical language to encode prescription instructions for medicines. Incorrect prescriptions by physicians can lead to errors and, thus, complications for the patients. Drug monographs provide detailed text instructions to warn about possible side-effects. However, reading with the needed attention the provided information is a very time-consuming activity. Glyphs assembled through graphic primitives such as shapes and colors are used in conjunction with icons to describe side effects or medical actions. Evaluation results showed that physicians understood $89 \%$ of the produced icons and were able to understand graphical sentences $1.8 \mathrm{x}$ faster than textual data. Encoding medical textual information in graphical icons or glyphs has also been studied by Müller et al. [20]. Patient case histories are encoded in a single glyph which reports the main diagnosis and an overview of the most recent events such as examinations and treatments. This information can also be presented at different detail levels, by accessing glyphs representing past events individually. These previous works use glyphs from a static viewpoint. Information is encoded into a glyph which then remains immutable. We wanted to go beyond this concept and, in our previous work [11], we explored the design space of a spatially distributed system that uses dynamic glyphs to support work activities in the medical domain.

Our goal was to investigate how best to model the data coming from activity-aware sources, so that by providing an always up-to-date graphical rendition of the quality of care through glyphs, real improvement could be attained. In that particular instance, we studied the different information categories that need to be considered when modelling a glyph: what information to present, how, where and when to present it. Key findings from this study prompted us to perform further research into the information space that can be portrayed by situated glyphs, the results of which we present in this paper. 

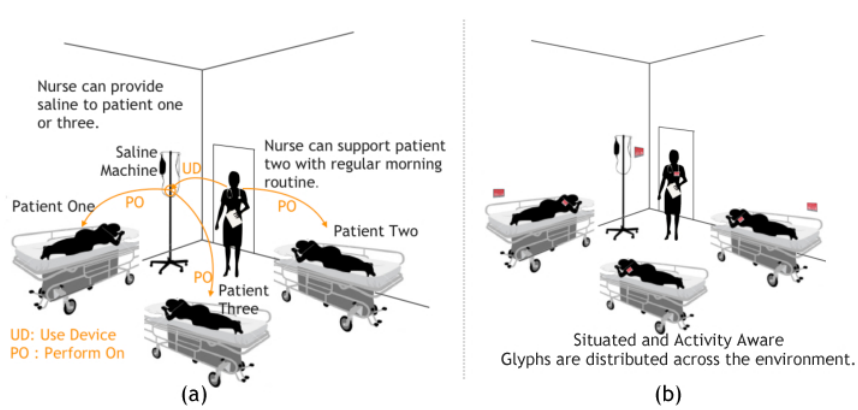

(b)

Fig. 1. A Hypothetical Nursing Home Scenario with and without Situated Glyphs

\section{Glyphs as Activity-Aware Visual Instructions for DyNAMic Work Environments}

In the field of information visualization, a glyph is a graphical object designed to convey multiple data [17]. Different parts of the representation or different visual attributes (e.g., shape, size, colour) are utilized to encode different values. One early example was shown by Chernoff [21] who represented multidimensional data through different attributes of human faces, e.g., a nose, eyes. In contemporary literature, researchers have used glyphs to represent different attributes of documents [22], for visualising software management data [23] or for visualization purposes in military command and control applications [24].

Due to their intrinsic capability of representing multiple variables with a single graphical representation, there are opportunities to explore the use of glyphs for exposing salient information in a subtle fashion in dynamic work places. Recent studies have shown that there is a clear need to present task-centric information in demanding work places, such as hospitals or industrial plants [8], [25]. Consider the situation depicted in Figure 1(a), where a nurse has multiple choices of activities with multiple patients and objects. She might decide to use the saline equipment with the patient one or three, or she might decide to support patient two. In all these cases, she needs information that matches her activity. Previous studies have shown that medical personnel would benefit most from having specific information available (e.g., guidelines) about their current activity, linked to relevant equipment and patients in this activity's context [8], [26].

To this end, we envision that glyphs provide an interesting design alternative to present real time in-situ information to support interleaved multiple activities involving multiple individuals and equipment in complex workplaces. In our previous work [11], we devised "Situated Glyphs" which are graphical units that are situated in time and space - they are visual representations of activities, and are adaptive, mobile and replaceable. Fig. 1(b) envisions the same situation as explained above, but here the environment is augmented with multiple situated glyphs. In this case when a nurse approaches an equipment or a patient to perform an activity, corresponding glyphs show the information that is relevant to that activity. One of the key functions of situated glyphs is to help people discover the activities that can be performed in a given space, at a given time with the devices and objects at hand.

There has been a lot of work on information provisioning through ambient displays - often embedded in interesting

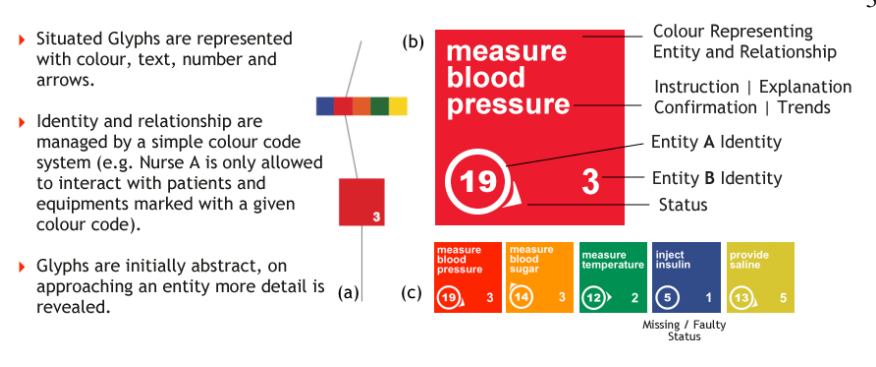

Fig. 2. An illustrative design of a situated glyph.

artistic objects or everyday artefacts, distributed across the environment and providing a constant stream of peripheral information [27]-[30]. Information presented via ambient displays is always interesting, sometimes useful but rarely vital. In contrast, our focus with situated glyphs is activity-centred. Our aim is to support (cognitive and physically) demanding real-world activities (such as nursing tasks in a hospital) by mapping visual representations of activity-specific information to physical environment using situated glyphs.

Our first glyph design (Figure 2) was created in close coordination with an expert designer, based on our requirements for situated glyphs. We have later adapted these designs into a prototype visual language following insights from the information visualization literature. The glyph in Figure 2(b) corresponds to a "red" coded nurse's activity of measuring blood pressure with a "red" coded patient numbered " 3 ", using a "red" coded blood monitoring device numbered "19" which is available in the south-east direction. This glyph design is adaptive and dynamically changes its content depending on the activity at hand and the context of the activity, i.e., glyphs are initially abstract, on approaching an individual or an object more detail is revealed as in Figure 2(a).

Previously, we identified [11] four different design cardinals for these situated glyphs: information affinity, representation density, spatial distribution and temporal distribution - which address content, appearance, placement, and timing aspects of situated glyphs. In this study, we seek to gain a better understanding of the design space for situated glyphs in the healthcare domain, and focus mainly on the first two design cardinals: information affinity and representation density. These describe what information should be presented to caregivers and how to present it. After all, to get conclusive answers on the other dimensions of the design space (i.e., when and where information should be presented), we should know first what information should be presented and through which means (both in terms of design and technology).

\section{What Information to Present and How: An Exploratory Study In a Psychogeriatrics Ward}

\section{A. Motivation}

To better understand how situated glyphs can be employed in the healthcare domain, a study was conducted at a medical facility which aimed to investigate what information should be presented to caregivers using situated glyphs and how this information should be visualized.

The study consisted of a one-day brainstorming workshop with nurses working in a psychogeriatrics ward. Psychogeriatrics is a subspecialty of psychiatry dealing with the study, 
prevention, and treatment of mental disorders in humans with old age, such as dementia. Besides providing primary care, nurses in this ward also spend a significant part of their time providing secondary care. This includes helping patients with their activities of daily living (e.g., a typical morning routine: morning hygiene, getting dressed and having breakfast), a greater emphasis on social contact with the patients, promoting and maintaining an individuals self-esteem and supporting patients in their social and psychological needs. The medical staff in this ward requires more - and at times more subtle - information than the traditional clinical information (e.g., medical history, vital signs, allergies), such as a profile of the patient's mental capabilities, how to best approach them, or their personal history (e.g., occupation, children, spouse). This need for detailed patient information motivated us to select this type of ward for gathering feedback on our ideas.

\section{B. Location and Participants}

The workshop took place at the psychogeriatrics ward of a care center in the province of Limburg, the Netherlands. The managing organization provides elderly care in this specific region, which besides these care centers also includes home care and housing. The psychogeriatrics ward where the study took place at that time cared for 55 patients, and employed about 60 staff members.

We recruited 7 participants from this ward (1 male and 6 female) for a focused brainstorming workshop. The group consisted of 6 nurses (the team lead and 5 other nurses), and 1 recreational therapist ${ }^{1}$ who also works closely with the patients and interacts with the nurses on a daily basis. Participants ranged from 23 to 58 years old, with a mean age of 42. In a demographic survey that was conducted before the start of the workshop, we also asked participants about their experience with technology such as computers and mobile phones, their responsibilities and experience in their current job. All participants reported owning a mobile phone. They rated their experience with computers and mobile phones as average, with a mean expertise of 3.29 for the former and 3.14 for the latter $(5=$ expert, $1=$ none $)$. On average, participants had 15.43 years of experience $(\tilde{x}=9.00, \sigma=10.94)$. We asked nurses about any other responsibilities they might have next to their main job. All but one nurse listed one or more additional responsibilities, ranging from being the personal caregiver for certain patients, taking care of planning and task allocation, to serving as one of the ward's incontinence officers.

\section{Method}

During the one-day workshop, nurses were asked to participate in brainstorm exercises about information needs and glyph design, as well as in open discussions about their day-to-day work activities, problems they encounter, and how situated glyphs could possibly be used to help them in their job. In order to make our ideas concrete, we demonstrated situated glyphs through technology probes, and explained our initial glyph designs.

\footnotetext{
${ }^{1}$ Recreational therapists use recreation as a way of promoting and maintaining an individuals self-esteem and social and psychological needs.
}

The workshop was organized in three separate sessions with breaks in-between. Before the start of the workshop, we introduced ourselves and explained our motivation and the general structure of the workshop to the participants. During the first session, participants introduces themselves to us and to the rest of the group. This was followed by a group discussion on their typical work day, and the main issues and difficulties they encounter in their job (asking questions such as: "What can go wrong and why?" or "How do you know what to do?").

In the second session, we asked participants to list different types of information that they need in their daily work activities. They were given paper index cards and were asked to write down as many different information types as possible, as shown in Figure 4(a-b). Afterwards, participants had to discuss each of the information types they wrote down in group and other participants were instructed to raise their hands when they wrote down the same information type (Figure 4(c)).

The third session started out with an explanation of the idea of situated glyphs, after which we showed participants the glyph designs and technology probes. Participants were introduced to the idea of having networked micro displays that could be distributed in different locations in the ward, such as in patient rooms or the supply room and could be interacted with using touch. As a proof of concept, we used iPod touch devices encased inside acrylic boxes, as shown in Fig. 3. The front panel of the box has a cut-out hole so that the iPod frame and part of its screen are hidden away. As a result, only a $4.5 \times 4.5 \mathrm{~cm}$ display area is visible. In this way, observers of the device are not led to assume that they are viewing the display of an iPod touch and will instead treat it as a "normal" screen. To illustrate different form factors, we also showed a small 51×30 mm OLED display module (Fig. 3). Participants were told that this form factor would enable glyph displays to be attached to objects or to be worn on the wrist like a watch.

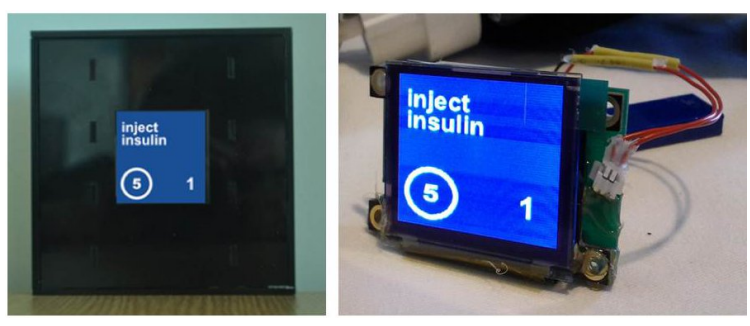

Fig. 3. The technology probes used during the workshop.

After this demonstration, we distributed a new set of index cards and instructed participants to show us how they would represent each of the information types they wrote down before. They were told to use whatever representation seemed right (e.g., symbols, icons, numbers, text, or combinations of these) (Fig. 4(d)). Participants then again discussed their designs in group, to reflect on which representation they deemed most appropriate.

The workshop was closed with an open group discussion, after which participants received a box of chocolates as a reward for their participation. We used video recordings, photographs and voice recordings for later analysis. The recordings were 


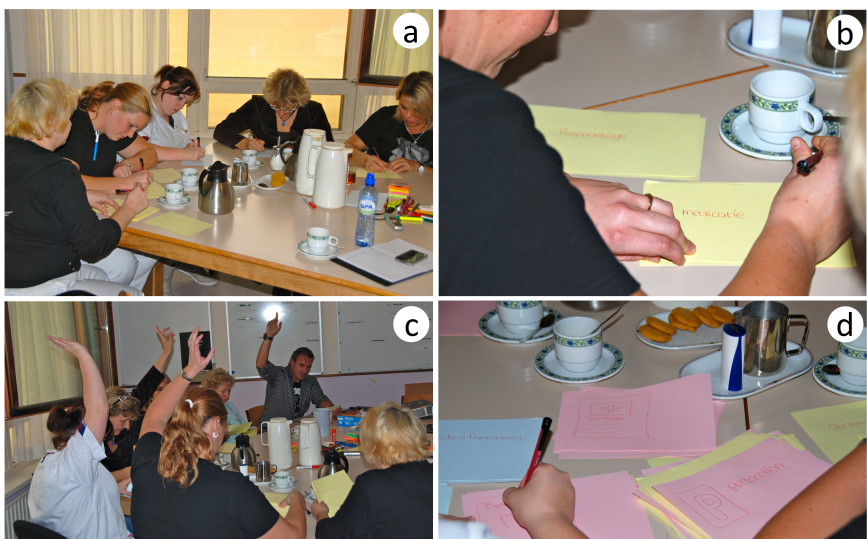

Fig. 4. (a-b) Participants writing down different types of information they require in their job on index cards; (c) afterwards, raising their hands to indicate that they wrote down the same information type; and (d) finally, designing glyphs for the information types they wrote down before.

transcribed and combined with the photographs, after which we collated the results and analysed the data. We used affinity diagrams to group items and discover patterns.

\section{Observations}

1) Daily Work Issues: When participants were asked about their day-to-day activities, two important findings emerged:

a) Interruptions: We asked about the kind of emergencies or unexpected problems that might arise during a typical day. They mentioned amongst others being called away, when they have to leave the patient alone. This could happen for several reasons: the telephone rings; another nurse needs assistance; they might have to assist the physician while doing a round of the ward; they might be notified of a triggered motion sensor on their pager which indicates that one of the patients has left their room. This indicates that nurses encounter many interruptions, and will benefit from having the right information available at the right time to be able to quickly return to a previous activity.

b) Information overload: Participants mentioned that their principal means of communication with colleagues is the patient's file. This is an all encompassing document which contains all relevant information specific to that patient, including important phone numbers, the patient's schedule, tasks that have to be performed and logs of care activities. However, nurses mentioned that even though this document contains all relevant information, they often don't have the entire file with them as it is too big. This means they have to run around a lot when they need additional information. Moreover, medical files also contain protocols specific to certain conditions that the patient suffers from such as bedsores. Although these protocols should in principle be known by the nurses, some conditions only occur rarely and it would thus be helpful to get a quick summary of this protocol to refresh their memory. Additionally, nurses said that they do not have the time to go through all information in a patient's file, and that it would thus be helpful to get relevant information when needed. These results indicate that nurses in this type of ward have to deal with an enormous information overload. We believe that situated glyphs could be used to help nurses obtain the right information at the right time in order to cope with this information overload, and would save them time so more time can be spent caring for the patients.

2) Information Types: The first brainstorming exercise resulted in 88 index cards (approximately 12 per participant). From the resulting group discussion and further analysis after the workshop, we distilled 28 unique information types. We eliminated duplicates, clustered similar information together and afterwards contacted the team lead again to verify our findings and understanding of the information types. Table I shows these 28 information types together with a short description and the number of participants that listed them in the brainstorm exercise. We noticed that many of the information types are specific to this type of ward or related to secondary care activities (e.g., strategies on how to approach a patient, and the patient's course of life).

3) Glyph Design: The glyph design brainstorm exercise resulted in 54 glyph designs, of which a few examples are shown in Fig 5. After analysing these designs, we were able to distinguish between different patterns of representations used by the nurses in their designs, as shown in Table II. There were a number of interesting observations: nurses used a variety of different representations in their designs; they mainly relied on two information presentation modalities (lists and schedules) for presenting more complex information; important or exceptional information was consistently indicated using exclamation marks; and information that logically belongs together was often represented within a single glyph.
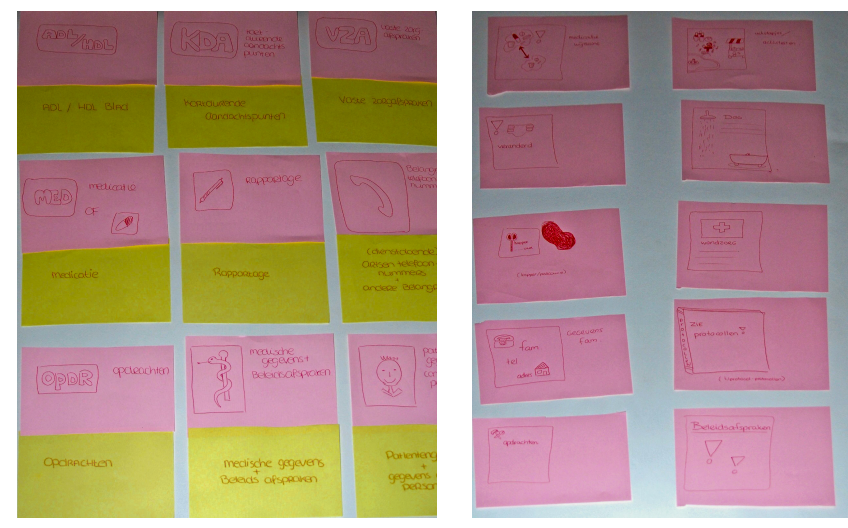

Fig. 5. Some examples of the glyphs that participants designed in the workshop. Note the use of both textual, iconic and symbolic information, as well as the use of domain-specific acronyms (e.g., ADL/HDL, KDA, VZA) or abbreviations (e.g., MED, OPDR). Participants frequently used exclamation marks to indicate important information.

\section{Discussion}

We concretise our findings in two ways. First, we present an updated glyph design, based on the information types and designs by the workshop participants. Secondly, we generalize our approach in the form of design guidelines which we feel can help designers to encode activity-centric information into situated glyphs for medical professionals. 


\begin{tabular}{|c|c|c|}
\hline Information Type & Amt. & Description \\
\hline Physician on Duty & 5 & Contact details of the primary physician currently on duty. \\
\hline Important Phone Numbers & 4 & Important numbers, such as those of the backup nurse and of the other wards. \\
\hline Protocols & 5 & $\begin{array}{l}\text { General protocols for certain conditions which are applicable to the patient (e.g., how to deal with and treat } \\
\text { bedsores, how to lift patients). }\end{array}$ \\
\hline Nutritional Information & 4 & Whether patients have specific nutritional requirements (e.g., a pureed diet). \\
\hline Patient and Family Data & 5 & Personal data about the patient and their family members (the main contact). \\
\hline Reporting & 4 & $\begin{array}{l}\text { Reports of a patient's current state or condition whenever a caregiver visits a patient (e.g., "Miss Doe was } \\
\text { particularly cheerful today"). }\end{array}$ \\
\hline Course of Life & 1 & $\begin{array}{l}\text { The patient's history: their occupation, whether they were married or had children, and social activities. This } \\
\text { information is used to improve conversations as patients suffering from dementia often return to their past. }\end{array}$ \\
\hline Daily Schedule & 3 & $\begin{array}{l}\text { A patient's daily schedule, including recreational activities, care giving appointments (e.g., physiotherapy) } \\
\text { and others (e.g., pedicure, hairdresser). }\end{array}$ \\
\hline Appointments & 5 & Includes medical and caregiving appointments, as well shower or bath days. \\
\hline Short-Term Attention Points & 4 & Temporary attention points (e.g., small injuries, the application of ointments). \\
\hline Approach Strategy & 4 & $\begin{array}{l}\text { Guidelines on how to approach a patient based on their behaviour (e.g., aggressiveness) and medical data } \\
\text { (e.g., hearing or visual impairment). }\end{array}$ \\
\hline Allergies & 2 & A patient's allergies and corresponding information (e.g., symptoms). \\
\hline $\begin{array}{l}\text { Activities of Daily } \\
\text { (ADL) }\end{array}$ & 4 & $\begin{array}{l}\text { Reports on how well patients can perform daily self-care activities (e.g., personal hygiene and grooming, } \\
\text { (un)dressing, bowel and bladder management). }\end{array}$ \\
\hline Assignments & 3 & $\begin{array}{l}\text { List of planned activities. Activities are concrete and specific to a patient with a date of entry and a due date, } \\
\text { but have not yet been assigned to staff members. }\end{array}$ \\
\hline Signal List & 6 & $\begin{array}{l}\text { A daily list of care activities that have to be performed to a certain patient. Activities are assigned to a } \\
\text { discipline or person (e.g., nurses, physician, physio). }\end{array}$ \\
\hline Medication & 6 & A list of medication that a patient uses including changes in medication. \\
\hline Clinical Picture & 2 & A summarized diagnosis of the patient's illnesses (e.g., Alzheimer's, diabetes) \\
\hline Resuscitation Policy & 2 & $\begin{array}{l}\text { Legal orders to repect a patient's wishes to not undergo CPR or advanced cardiac life support if their heart } \\
\text { were to stop or they were to stop breathing. }\end{array}$ \\
\hline Wound Care & 2 & $\begin{array}{l}\text { Reports on the evolution of wounds (e.g., redness, swelling) and caregiving activities for treating those wounds } \\
\text { (e.g., cleaning, dressing). }\end{array}$ \\
\hline Incidents & 3 & Reports of occurred incidents (e.g., medication errors, aggression, falls). \\
\hline Evaluation & 1 & $\begin{array}{l}\text { Evaluation of previously reported events or conditions (e.g., short-term attention points that fail to improve } \\
\text { such as frequent falls) and the corresponding action to take (e.g., an appointment with the physiotherapist). }\end{array}$ \\
\hline Incontinence Changes & 3 & Evolution of a patient's incontinence and the required incontinence material. \\
\hline Materials & 2 & A list of required materials (e.g., incontinence pads) per patient. Answers: "Who needs what and where?" \\
\hline Life Plan Conversation & 2 & $\begin{array}{l}\text { Reports of periodic meetings with the physician, family and caregivers where the patient's evolution and any } \\
\text { incidents are discussed. Will also include policies (e.g., whether patients need to be fixated, resuscitation). }\end{array}$ \\
\hline Family Participation & 1 & Details of participation by family members in caring for the patient. \\
\hline Patient Check-up & 2 & Diagnosis by physicians and other specialists (e.g., the physiotherapist, ergotherapist, or psychologist). \\
\hline Behaviour Profile & 2 & Profile of the patient's behaviour including recent changes (e.g., agressive fits). \\
\hline Bodily Functions & 2 & Includes vital signs such as the patients blood pressure but also stool patterns. \\
\hline
\end{tabular}

TABLE I

THE DIFFERENT INFORMATION TYPES IDENTIFIED IN THE WORKSHOP.

\section{A. Updated Glyph Design}

We have revised our original glyph design to account for the information types identified in the workshop (see Table I) and the notations participants used in their designs (see Table II). Our original design focused mostly on primary (medical) care activities, such as providing medication or checking a patient's vital signs. During the workshop, nurses expressed a need for more detailed information, such as a patient's daily schedule, as well as less activity-centric information, such as their level of autonomy with respect to Activities of Daily Living (ADL). Three example glyphs that use our revised design are shown in Figure 6. The new glyphs are characterized by a greater emphasis on information types deemed important by the nurses such as daily schedules or secondary care activities, and make better use of the notations they employ everyday (e.g., acronyms for disciplines, exclamation marks).

Not all information types in Table I are activity-specific (e.g., Course of Life). In future work, we feel it is necessary to get a better understanding of how to best present this type of information to caregivers, without overwhelming or distracting them. One approach could be to allow caregivers to interact

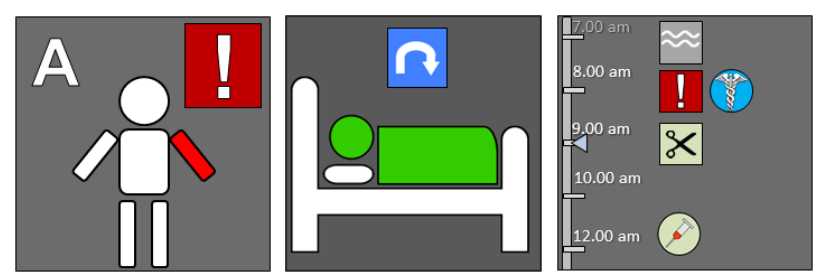

Fig. 6. Our updated design based on the results from the workshop. Note the use of acronyms (e.g., A for "Arts", which is doctor in Dutch), time schedules, exclamation marks to indicate important or exceptional information, the caduceus symbol, and the inclusion of secondary care activities (e.g., the scissors indicating an appointment with the hairdresser).

with the glyphs to quickly request specific information (such as the patient's primary contact, in case a family member needs to be contacted).

\section{B. Design Guidelines}

The overarching goal of situated glyphs is to raise awareness of events that have happened or will happen and provide guidance on how to attend them. Based on our findings, we 


\begin{tabular}{|c|c|c|}
\hline Representation & Example & Usage \\
\hline Single Letters & $\frac{\text { Disciplines }}{|F|}$ & $\begin{array}{l}\text { Categories which are easily distinguishable using only a single letter, such as } \\
\text { disciplines (e.g., E: ergo, PH/F: physio, P: psychologist). }\end{array}$ \\
\hline Symbols & 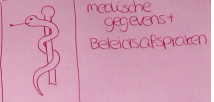 & $\begin{array}{l}\text { Well-known symbols or icons, such as the caduceus symbol indicating medicine } \\
\text { (or medical data in this particular case). }\end{array}$ \\
\hline Acronyms & & $\begin{array}{l}\text { Specific acronyms with which nurses are familiar and which are also used in reports } \\
\text { (e.g., ADL). }\end{array}$ \\
\hline Abbreviations & medicatie & $\begin{array}{l}\text { Well-known abbreviations that nurses often use as a short-hand in reports or for } \\
\text { documentation purposes (e.g., MED for medication). }\end{array}$ \\
\hline Lists and Tables & & $\begin{array}{l}\text { Various purposes, such as lists of incidents (in this case), tasks, short-term attention } \\
\text { points, materials, phone numbers, etc. }\end{array}$ \\
\hline Schedules & & $\begin{array}{l}\text { Various purposes, such as a patient's daily schedule, specific appointments, when } \\
\text { to provide medication. Needs to draw attention to events that deviate from the } \\
\text { standard routine (e.g., changes in when medication is administered). }\end{array}$ \\
\hline Exclamation Marks & veranderd & $\begin{array}{l}\text { Exceptions, changes to the regular routines or important information (e.g., in this } \\
\text { case a change in incontinence material). }\end{array}$ \\
\hline Pictures & $\begin{array}{l}\text { patent } \\
\text { gegevens. } \\
\text { comtact } \\
\text { personen }\end{array}$ & $\begin{array}{l}\text { Representing patients with pictures was deemed useful in general, but especially } \\
\text { for new employees or supporting nurses who only work at the ward on a temporary } \\
\text { basis. }\end{array}$ \\
\hline Multi-Dimensional & & $\begin{array}{l}\text { Several data that together form a logical unit (e.g., bodily functions, such as blood } \\
\text { pressure, pulse, weight, stool patterns). }\end{array}$ \\
\hline
\end{tabular}

TABLE II

THE DIFFERENT TYPES OF REPRESENTATIONS USED IN THE GLYPH DESIGNS AT THE WORKSHOP.

distilled a number of principles we feel are important in the design of situated glyphs for healthcare applications:

1) Design for a dynamic work environment: Since caregivers typically have to deal with many interruptions, glyphs need to immediately communicate their intended meaning to caregivers so that they can be aware of their purpose with no more than a few glances. Glyphs should strike a delicate balance between providing enough information and still being easy to read. Participants also expressed concerns about the readability of information on small displays, especially for older staff.

2) Inform, don't distract: Glyphs should not distract observers, whether those are patients or caregivers. This means designers should be cautious to use animations or sounds to draw the attention. Especially patients who suffer from mental illnesses (e.g., dementia), might be confused or distracted by attention-grabbing displays.

3) Respect the patient's privacy: Glyphs need to hide sensible information to visitors and other patients, if possible. For example, a glyph could show that a patient needs to be treated with a specific type of medicine or undergo an examination, whose exact type is known to the nurse, thus avoiding the need to reveal the specific kind. Another possibility is to use identification technology (e.g., NFC) to only show information to authorized personnel. During the workshop, however, participants mentioned that it is ultimately their own responsibility to make sure the patient's privacy is respected.

4) Contextualize: It is important for a solution that employs situated glyphs to take into account the environment in which it will be deployed. There is no "one size fits all" approach, as the hospital staff will, 
to a large extent, be accustomed to local practices. Therefore, the design of a glyph-based system should take these practices into account and not force caregivers to use a new way of working. As mentioned before, during the workshop, we observed how participants used abbreviations that they are familiar with and how they organize information in the form of structures that they are accustomed to, such as schedules or lists. This also means that glyphs should not be overly detailed, as the hospital staff is assumed to know their procedures.

However, finding a suitable way to encode information into a "glyph" is not a trivial problem. An ideal glyph design would have to be based on a consistent grammar, be able to model several different information types and provide extensibility should new needs arise. The information space to model should cover different types of activities, notifications, and events; it should also be appropriate for the target audience considered, final display devices, etc. This, in turn, leads to a vast solution space and makes it difficult to establish whether a particular design is the most appropriate.

In this paper, we have investigated the application of situated glyphs in healthcare. Situated glyphs are visual, dynamic representations of activities that assist caregivers in discovering activities that can be performed in a given situation. We organized a workshop to investigate the information space to encode into glyphs, and gathered examples of glyph designs through participatory design with medical professionals. Based on these results, we have shown concrete examples of glyph designs that take these findings into account, and have provided a number of design guidelines which we feel can help designers to encode activity-centric information into situated glyphs for healthcare professionals. When deploying situated glyphs in healthcare facilities, we recommend using a usercentered design approach to ensure that the implementation of situated glyphs fits with the work practices of the caregivers.

While our findings are not necessarily generalizable to other healthcare environments, participants stated that they expected situated glyphs to be useful in other types of wards as well (e.g., an intensive care unit). In general, we expect situated glyphs to work well for environments in which workers have to deal with frequent interruptions and need access to detailed information while performing their daily duties.

\section{REFERENCES}

[1] A. M. Epstein, "Paying for performance in the United States and abroad," The New England journal of medicine, vol. 355, no. 4, pp. 406-408, 2006.

[2] S. M. Campbell, D. Reeves, E. Kontopantelis, B. Sibbald, and M. Roland, "Effects of Pay for Performance on the Quality of Primary Care in England," New England Journal of Medicine, vol. 361, no. 4, pp. 368-378, 2009.

[3] P. K. Lindenauer, D. Remus, S. Roman, M. B. Rothberg, E. M. Benjamin, A. Ma, and D. W. Bratzler, "Public Reporting and Pay for Performance in Hospital Quality Improvement," New England Journal of Medicine, vol. 356, no. 5, pp. 486-496, 2007.

[4] M. Tentori, D. Segura, and J. Favela, "Monitoring Hospital Patients Using Ambient Displays," in Mobile Health Solutions for Biomedical Applications, P. Olla and J. Tan, Eds. IGI Global, 2009, pp. 143-158.

[5] N. Bricon-Souf and C. R. Newman, "Context awareness in health care: a review." International journal of medical informatics, vol. 76, no. 1, pp. 2-12, Jan. 2007.
[6] G. Chen and D. Kotz, "A Survey of Context-Aware Mobile Computing Research," Nov. 2000.

[7] J. Bardram and H. Christensen, "Pervasive Computing Support for Hospitals: An overview of the Activity-Based Computing Project," IEEE Pervasive Computing, vol. 6, no. 1, pp. 44-51, Jan. 2007.

[8] J. E. Bardram, "A novel approach for creating activity-aware applications in a hospital environment," in Proc. INTERACT '09. Springer, 2009. pp. 731-744.

[9] J. Bardram, "Applications of context-aware computing in hospital work," in Proc. SAC '04. ACM Press, Mar. 2004, pp. 1574-1579.

[10] J. Favela, M. Tentori, L. A. Castro, V. M. Gonzalez, E. B. Moran, and A. I. Martínez-García, "Activity Recognition for Context-aware Hospital Applications: Issues and Opportunities for the Deployment of Pervasive Networks," Mobile Networks and Applications, vol. 12, no. 2-3, pp. 155-171, Jul. 2007.

[11] F. Kawsar, J. Vermeulen, K. Smith, K. Luyten, and G. Kortuem, "Exploring the design space for situated glyphs to support dynamic work environments," in Proc. Pervasive '11, ser. Lecture Notes in Computer Science, K. Lyons, J. Hightower, and E. Huang, Eds. Springer Berlin / Heidelberg, 2011, vol. 6696, pp. 70-78.

[12] J. E. Bardram, T. R. Hansen, and M. Soegaard, "AwareMedia," in Proc. of $C S C W$ '06. ACM Press, Nov. 2006, pp. 109-118.

[13] D. Segura, J. Favela, and T. Mónica, "Sentient Displays in Support of Hospital Work," in Advances in Intelligent and Soft Computing, ser. Advances in Soft Computing, J. M. Corchado, D. I. Tapia, and J. Bravo, Eds. Berlin, Heidelberg: Springer Berlin Heidelberg, 2009, vol. 51, pp. $103-111$.

[14] T.-F. Kummer, M. Bick, and R. Gururajan, "Acceptance problems of ambient intelligence and mobile technologies in hospitals in India and Germany," in Proc. ECIS '09, 2009.

[15] S. Spiekermann, User control in ubiquitous computing: design alternatives and user acceptance. Shaker, May 2008.

[16] A. L. Friedman, S. R. Geoghegan, N. M. Sowers, S. Kulkarni, and R. N. Formica, "Medication Errors in the Outpatient Setting," Archives of Surgery, vol. 142, no. 3, pp. 278-283, 2007.

[17] C. Ware, Information Visualization: Perception for Design. San Francisco, CA, USA: Morgan Kaufmann Publishers Inc., Apr. 2004.

[18] T. Ropinski and B. Preim, "Taxonomy and Usage Guidelines for Glyphbased Medical Visualization," in Simulation and Visualization 2008 SimVis 2008, 2008, pp. 121-138.

[19] J.-B. Lamy, C. Duclos, A. Bar-Hen, P. Ouvrard, and A. Venot, "An iconic language for the graphical representation of medical concepts," BMC Medical Informatics and Decision Making, vol. 8, no. 1, p. 16, 2008.

[20] H. Müller, S. Sauer, K. Zatloukal, and T. Bauernhofer, "Interactive Patient Records," Proc. IV '10, pp. 252-257, 2010.

[21] H. Chernoff, "The use of faces to represent points in k-dimensional space graphically," Journal of the American Statistical Association, pp. $361-368,1973$

[22] T. Mann, "Visualization of www-search results," in Proc. DEXA '99, 1999, pp. 264-268.

[23] M. C. Chuah and S. G. Eick, "Information rich glyphs for software management data," IEEE Comput. Graph. Appl., vol. 18, no. 4, pp. 2429, Jul. 1998.

[24] R. Whitaker and G. Thomas-Meyers, "Knowledge glyphs: visualization theory development to support c2 practice," in Proc. Winter Simulation Conference '06, 2006, pp. 1203-1209.

[25] C. Heyer, "Investigations of ubicomp in the oil and gas industry," in Proc. Ubicomp '10. New York, NY, USA: ACM, 2010, pp. 61-64.

[26] J. E. Bardram, T. R. Hansen, M. Mogensen, and M. Søgaard, "Experiences from real-world deployment of context-aware technologies in a hospital environment," in Proc. Ubicomp '06, 2006, pp. 369-386.

[27] C. Wisneski, H. Ishii, A. Dahley, M. G. Gorbet, S. Brave, B. Ullmer, and P. Yarin, "Ambient displays: Turning architectural space into an interface between people and digital information," in Proc. CoBuild '98. London, UK, UK: Springer-Verlag, 1998, pp. 22-32.

[28] N. Jafarinaimi, J. Forlizzi, A. Hurst, and J. Zimmerman, "Breakaway: an ambient display designed to change human behavior," in CHI EA '05. New York, NY, USA: ACM, 2005, pp. 1945-1948.

[29] Hallnäs, Lars and Redström, Johan, "Slow Technology Designing for Reflection," Personal Ubiquitous Comput., vol. 5, no. 3, pp. 201-212, Jan. 2001.

[30] Z. Pousman and J. Stasko, "A taxonomy of ambient information systems: four patterns of design," in Proc. AVI '06. New York, NY, USA: ACM, 2006, pp. 67-74. 